\title{
Three-Dimensional Combined Diversity Coding and Error Control Coding: Code Design and Diversity Analysis
}

\author{
Jinsong $\mathrm{Wu}^{\dagger}$, Pei Xiao ${ }^{\dagger \dagger}$, Qingchun Chen ${ }^{\dagger \dagger}$, Steven D. Blostein ${ }^{\S}$ \\ ${ }^{\dagger}$ Alcatel Shanghai Bell Company Ltd. \\ Pudong Jinqiao, Shanghai 201206, China \\ E-mail:wujs@ieee.org \\ ${ }^{\dagger \dagger}$ Centre for Communication Systems Research (CCSR) \\ University of Surrey, Guildford, Surrey, GU2 7XH, United Kingdom \\ E-mail: p.xiao@surrey.ac.uk \\ ${ }^{\dagger \dagger}$ Key Lab of Information Coding and Transmission \\ Southwest Jiaotong University, Chengdu 610031, China \\ E-mail: qcchen@swjtu.edu.cn \\ ${ }^{\S}$ Department of Electrical and Computer Engineering \\ Queen's University, Kingston, Ontario, K7L 3N6, Canada \\ E-mail: steven.blostein@queensu.ca
}

\begin{abstract}
This paper investigates joint complex diversity coding (CDC) and error control coding (ECC) to increase diversity gains across multiple antennas, OFDM blocks, and OFDM subcarriers. A general diversity analysis for joint CDC and ECC based space-time-frequency codes (STFCs) is provided. The mapping designs from ECC to CDC are crucial for efficient exploitation of the diversity potential. This paper provides and proves a sufficient condition of full diversity construction with joint 3 D CDC and ECC, bit-interleaved coded complex diversity coding (BICCDC) and symbol-interleaved coded complex diversity coding (SICCDC). A multi-stream architecture is also introduced to reduce the complexity and latency of the decoding process.
\end{abstract}

\section{INTRODUCTION}

Space-time coding (STC) [?, ?,?] has emerged as one of the most promising technologies for high data rate and high quality multiple-input multiple-output (MIMO) wireless communications. However, in frequency-selective channels, STC cannot exploit available frequency diversity in MIMO orthogonal frequency division multiplexing (OFDM) systems. A challenging problem in wideband MIMO system design is to develop new high rate coding schemes to efficiently exploit all the diversity available across space, time and frequency domains. To this end, the design of space-time-frequency coding (STFC) has been recently investigated in $[?, ?, ?]$. We introduce a general terminology, complex diversity coding (CDC) which summarizes existing diversity coding approaches using complex conversion. Space-time-frequency codes may be categorized into different integrations of CDC and ECC. Basically, CDC reforms conventional constellation based signals into new form of diversity signals through complex transformation. Note that 1 ) for single-input single-output communications, a general term for complex coding is signal space diversity [?]; 2) for twodimensional channels, such as space time MIMO channels, a commonly used term for complex coding is linear dispersion codes (LDC). However, LDC was originally designed to optimize mutual information between transmitted and received signals [?], rather than utilize diversity criteria. In this paper, the concept of CDC is used to stress the diversity properties of complex coding approaches, especially for those with more than one physical dimension.

Although high-rate 3-dimensional (3-D) CDC have been proposed in [?,?], joint 3-D CDC and ECC has not been investigated and analyzed. In order to mitigate the fading effects, practical wireless systems often employ some forms of error correction code (ECC). Unlike the previous analyses for pure 3D CDC systems presented in [?], this paper provides a general diversity analysis for systems with joint 3-D CDC and ECC.

We also incorporate a joint lower complexity 3-D CDC and multi-stream ECC STFC system and compare its performance with that of single-stream systems.

The notation used in this paper is summarized as follows: $(\cdot)^{\mathcal{T}}$ stands for matrix transpose, $(\cdot)^{\mathcal{H}}$ stands for matrix transpose conjugate, $E[\cdot]$ stands for expectation operation, $j$ is the square root of $-1, \mathbf{I}_{K}$ denotes identity matrix of size $K \times K$, $\mathbf{0}_{M \times N}$ denotes zero matrix of size $M \times N, \mathbf{A} \otimes \mathbf{B}$ denotes Kronecker (tensor) product of matrices $\mathbf{A}$ and $\mathbf{B},[\mathbf{A}]_{a, b}$ denotes the $(a, b)$ entry of matrix $\mathbf{A}$, and $\operatorname{diag}(\cdot)$ transforms the argument from a vector to a diagonal matrix, and $\operatorname{vec}(\mathbf{X})$ denotes $\operatorname{vec}(\mathbf{X})=\left[\left[[\mathbf{X}]_{:, 1}\right]^{\mathcal{T}}, \ldots,\left[[\mathbf{X}]_{:, N}\right]^{\mathcal{T}}\right]^{\mathcal{T}}$, where matrix $\mathrm{X}$ is of size $M \times N$.

\section{SYSTEM MODEL}

A. Space-time-frequency block (STFB) and space-timefrequency code (STFC)

We consider a MIMO-OFDM system with $N_{t}$ transmit antennas, $N_{r}$ receive antennas, and a block of $N_{c}$ OFDM subcarriers per antenna. Channel coefficients are assumed to be 
constant within one OFDM block. However, the channel coefficients change from block to block, and they are assumed to be statistically independent among different OFDM blocks. The proposed system could thus be used in dynamic environments. One 3-D CDC-based STFC codeword contains $D$ space-timefrequency blocks (STFB), each of which is of size $N_{t} \times N_{F} \times T$, i.e., across $N_{t}$ transmit antennas $N_{F}$ subcarriers and T OFDM blocks, where $N_{C}=D N_{F}$. The data sequence is modulated using complex-valued symbols $\alpha_{q}+i \beta_{q}$, chosen from an arbitrary constellation (e.g., r-PSK or r-QAM). One STFB, denoted by $\mathbf{S}_{S T F B}$, can be written in matrix form as

$$
\mathbf{S}_{S T F B}=\sum_{q=1}^{Q}\left(\alpha_{q} \mathbf{A}_{q}+j \beta_{q} \mathbf{B}_{q}\right) .
$$

where $\mathbf{A}_{q} \in \mathbb{C}^{N_{T} \times N_{F} T}$ and $\mathbf{B}_{q} \in \mathbb{C}^{N_{T} \times N_{F} T}$ are dispersion matrices for the real and imaginary parts of source signals. Equation (1) may be considered as a 3-dimensional formulation of linear dispersion codes [?], and can be reformulated as follows:

1) if $\mathbf{A}_{q} \neq \mathbf{B}_{q}$,

$$
\operatorname{vec}\left(\mathbf{S}_{S T F B}\right)=\mathbf{G}_{S T F B}^{v e c} \theta
$$

where

$$
\begin{aligned}
& \mathbf{G}_{S T F B}^{v e c} \\
& =\left[\operatorname{vec}\left(\mathbf{A}_{1}\right), \ldots, \operatorname{vec}\left(\mathbf{A}_{Q}\right), j \operatorname{vec}\left(\mathbf{B}_{1}\right), \ldots, j \operatorname{vec}\left(\mathbf{B}_{Q}\right)\right] \\
& \theta=\left[\alpha_{1}, \ldots, \alpha_{Q}, \beta_{1}, \ldots, \beta_{Q}\right]^{\mathcal{T}} .
\end{aligned}
$$

2) if $\mathbf{A}_{q}=\mathbf{B}_{q}$,

$$
\operatorname{vec}\left(\mathbf{S}_{S T F B}\right)=\mathbf{G}_{S T F B} \mathbf{s},
$$

where

$$
\begin{aligned}
& \mathbf{G}_{S T F B}=\left[\operatorname{vec}\left(\mathbf{A}_{1}\right), \ldots, \operatorname{vec}\left(\mathbf{A}_{Q}\right)\right] \\
& \mathbf{s}=\left[s_{1}, \ldots, s_{Q}\right]^{\mathcal{T}} .
\end{aligned}
$$

We define the coding rate of CDC-based STFC as $R^{\text {sym }}=$ $\sum_{i=1}^{D} Q_{i} /\left(N_{t} N_{c} T\right)$ [?], where $Q_{i}$ is the number of source symbols encoded in the $i$-th STFB. In our simulations, we apply rate-one full diversity $\mathrm{CDC}$ based STFCs proposed in [?], and these codes satisfy $\mathbf{A}_{q}=\mathbf{B}_{q}$.

\section{B. Frequency domain system model and structure}

Consider one joint CDC-ECC STFC block across $K$ 3-D CDC based STFC codewords. The baseband frequency domain signal for the $i$-th STFB within the $k$-th STFC can be written as

$$
\mathbf{y}^{(i, k)}=\sqrt{\frac{\rho}{N_{t}}} \mathbf{H}_{S T F B}^{(i, k)} \mathbf{G}_{S T F B} \mathbf{s}^{(i, k)}+\mathbf{n}^{(i, k)},
$$

where $\mathbf{H}_{S T F B}^{(i, k)}$ is the corresponding frequency domain channel matrix of size $N_{r} N_{F} T \times N_{t} N_{F} T$. Both vectors $\mathbf{y}^{(i, k)}$ and $\mathbf{n}^{(i, k)}$ are of size $N_{r} N_{F} T$, and they are the frequency domain received signal and additive complex Gaussian noise vectors, respectively. The source signal vector $\mathbf{s}^{(i, k)}$ is of size $N_{t} N_{F} T$. The channel matrix $\mathbf{H}_{S T F B}^{(i, k)}$ is formed as

$$
\mathbf{H}_{S T F B}^{(i, k)}=\operatorname{diag}\left(\mathbf{H}_{S T F B}^{(i, 1, k)}, \ldots, \mathbf{H}_{S T F B}^{(i, T, k)}\right),
$$

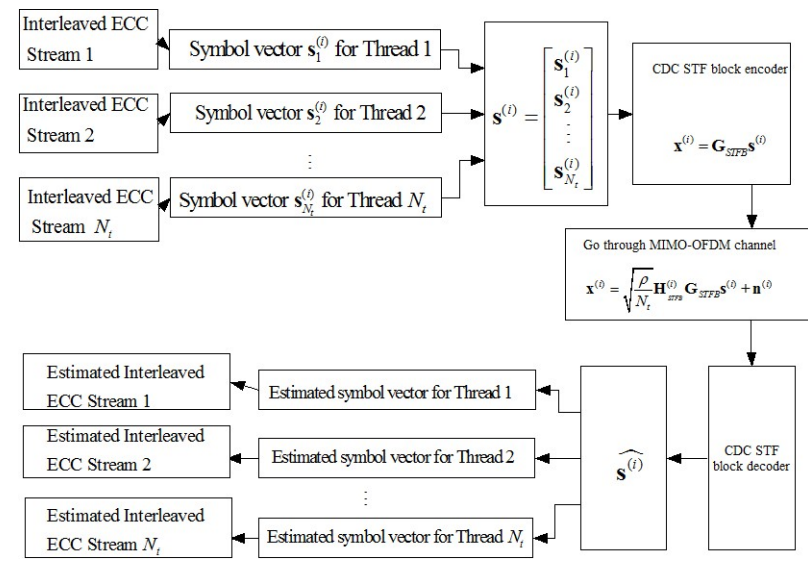

Fig. 1. Structure of Multi-stream joint CDC and ECC STFC

where $\mathbf{H}_{S T F B}^{(i, t, k)}=\operatorname{diag}\left(\mathbf{H}_{S T F B\left(p_{1}\right)}^{(i, t, k)}, \ldots, \mathbf{H}_{S T F B\left(P_{N_{F}}\right)}^{(i, t, k)}\right)$, $\mathbf{H}_{S T, k)}^{(i, t, k)}$ of size $N_{r} \times N_{t}$ is the frequency domain MIMO ${ }^{S}$ channel matrix for the $p$-th subcarrier, $t$-th OFDM block, $i$-th STFB, $k$-th CDC based STFC $\left(i=1 \cdots D, t=1 \cdots T, n_{F}=1 \cdots N_{F}, k=1 \cdots K\right)$. $\left\{p_{1}^{(i)}, \ldots, p_{N_{F}}^{(i)}\right\}$ is the subcarrier set chosen for the $i$-th CDC encoded STF block. For uniform power delay profile, the subcarrier set is chosen using even spacing over the whole subcarrier index space from 1 to $N_{C}$.

As shown in Fig. 1, the ECC coded streams are first interleaved with random interleaver, and mapped into complex source symbols, which are subsequently encoded into CDC based STFCs. It is assumed that one set of ECC streams for one joint CDC and ECC block is across $K$ STFCs and $N_{a}$ STFBs within one STFC. Fig. 2 illustrates one example of the proposed system structure, which is used in the simulations, where $D=8, N_{r}=N_{t}=2, T=2, N_{F}=4, N_{a}=4, N_{C}=$ $D N_{F}=32$ in one STFC. Note that there are 4 STFCs over time, and the each STFC consists of 8 STFBs. $\mathbf{G}_{S T F B}$ is a unitary matrix of size $16 \times 16$, and $\mathbf{s}^{(i)}=\left[\left[\mathbf{s}_{1}^{(i)}\right]^{\mathcal{T}},\left[\mathbf{s}_{2}^{(i)}\right]^{\mathcal{T}}\right]^{\mathcal{T}}$, where $\mathbf{s}_{m}^{(i)}, m=1,2$, is the source symbol vector for the $m$-th thread of the $i$-th STFB. In this work, the employed ECCs are convolutional codes, the mapping scheme of which is shown as follows:

$$
\left[\begin{array}{ccc}
\operatorname{STFB}_{1}^{(1)} & \cdots & \operatorname{STFB}_{1}^{(4)} \\
\vdots & \ddots & \vdots \\
\operatorname{STFB}_{4}^{(1)} & \cdots & \operatorname{STFB}_{4}^{(4)} \\
\operatorname{STFB}_{5}^{(1)} & \cdots & \operatorname{STFB}_{5}^{(4)} \\
\vdots & \ddots & \vdots \\
\operatorname{STFB}_{8}^{(1)} & \cdots & \operatorname{STFB}_{8}^{(4)}
\end{array}\right]
$$

where $\mathrm{STFB}_{i}^{(r)}$ stands for the $i$-th STFB in the $r$-th STFC. In this example, only one convolutional code is encoded in each block of joint CDC and ECC, as shown in each dashed rectangular area in Fig. 2. Iterative decoding may be applied within each block. Two joint CDC-ECC blocks are shown in the figure: the first one is across $\operatorname{STFB}_{i}^{(r)}, i=1, \ldots, 4, r=1, \ldots, 4$; the second one is across $\operatorname{STFB}_{i}^{(r)}, i=5, \ldots, 8, r=1, \ldots, 4$.

\section{Encoding matrix for CDC based STFC}

Assuming that frequency selective channel order $L$ is 1 (2tap channel) and $N_{F}=N_{t}(L+1)$. For $N_{t}=2, T=2$, the 


$$
\mathbf{S}_{S T F B}=\left[\begin{array}{cccccccc}
X_{1}(1) & \varphi X_{2}(2) & X_{1}(3) & \varphi X_{2}(4) & X_{1}(5) & \varphi X_{2}(6) & X_{1}(7) & \varphi X_{2}(8) \\
\varphi X_{2}(1) & X_{1}(2) & \varphi X_{2}(3) & X_{1}(4) & \varphi X_{2}(5) & X_{1}(6) & \varphi X_{2}(7) & X_{1}(8)
\end{array}\right],
$$

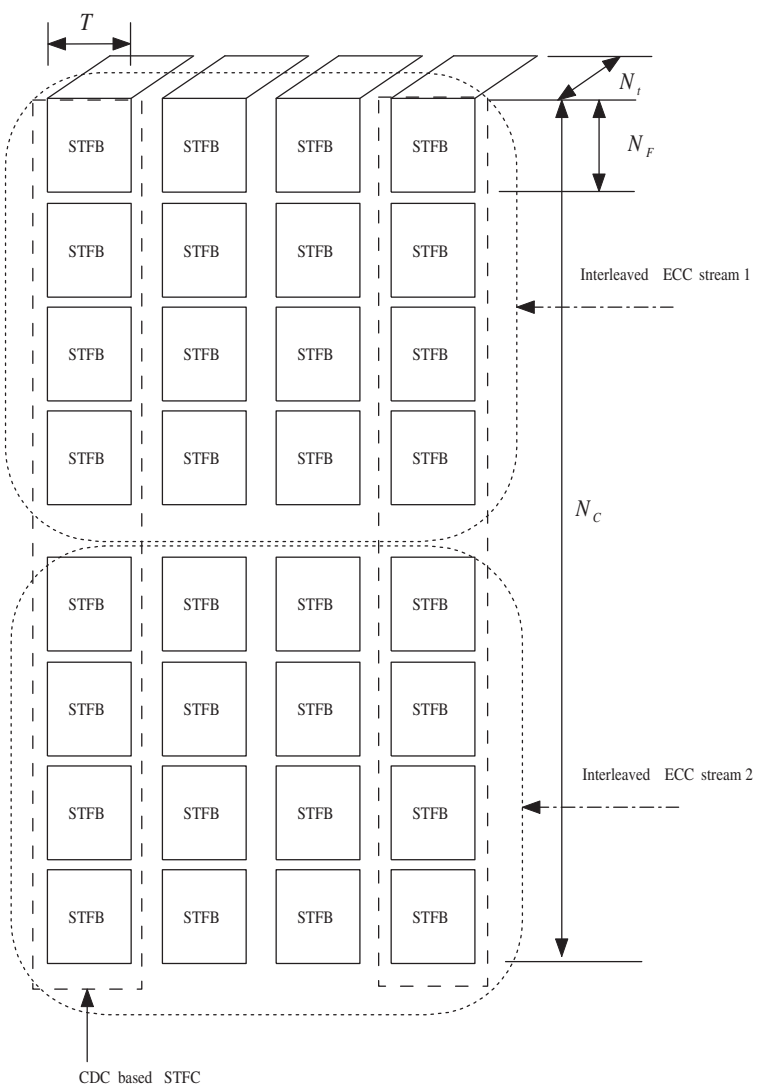

Fig. 2. The structure of the STFC code.

STFB expressed by Equ. (32) in [?] can be reformed as shown in (5) where

$$
\begin{aligned}
\mathbf{X}_{m} & =\left[X_{m}(1), \ldots, X_{m}(8)\right]^{\mathcal{T}}=\Theta\left[s_{m}(1), \ldots, s_{m}(8)\right]^{\mathcal{T}} \\
& =\Theta \mathbf{s}_{m}
\end{aligned}
$$

In (6), $\mathbf{s}_{m}=\left[s_{m}(1), \ldots, s_{m}(8)\right]^{\mathcal{T}}$ is the source symbol vector for the $m$-th thread of each STFB, and

$$
\Theta=\mathbf{F}_{M} \operatorname{diag}\left(1, \alpha, \ldots, \alpha^{M-1}\right),
$$

where $\mathbf{F}_{M}$ is $M \times M$ discrete Fourier transform matrix, $\alpha=$ $\exp (j 2 \pi /(4 M)), \theta=\alpha$, and $\varphi=\theta^{1 / 2}$, and $M=\frac{N_{F}}{N_{t}} N_{t} T=$ 8. The corresponding encoding matrix can now be written as

$$
\mathbf{G}_{S T F B}=\left[\begin{array}{c}
{\left[\boldsymbol{\Omega}^{0} \mathbf{U}\right] \otimes\left[\mathbf{e}_{1}^{(M)} \Theta\right]} \\
{\left[\mathbf{\Omega}^{1} \mathbf{U}\right] \otimes\left[\mathbf{e}_{2}^{(M)} \Theta\right]} \\
\vdots \\
{\left[\boldsymbol{\Omega}^{M-1} \mathbf{U}\right] \otimes\left[\mathbf{e}_{M}^{(M)} \Theta\right]}
\end{array}\right],
$$

where $\boldsymbol{\Omega}=\left[\begin{array}{ll}0 & 1 \\ 1 & 0\end{array}\right], \boldsymbol{\Omega}^{0}=\left[\begin{array}{ll}1 & 0 \\ 0 & 1\end{array}\right], \mathbf{U}=\left[\begin{array}{ll}1 & 0 \\ 0 & \varphi\end{array}\right]$, and $\mathbf{e}_{n}^{(M)}$ of size $1 \times M$ is an all-zero row vector except its value is 1 at the $n$-th position. The matrix $\mathbf{G}_{S T F B}$ is unitary, which can be proved as follows:

$$
\begin{aligned}
& {\left[\mathbf{G}_{\text {STFB }}\right]^{\mathcal{H}} \mathbf{G}_{S T F B}} \\
& =\sum_{n=1}^{M}\left[\left[[\mathbf{U}]^{\mathcal{H}}\left[\boldsymbol{\Omega}^{n-1}\right]^{\mathcal{H}}\right] \otimes\left[[\Theta]^{\mathcal{H}}\left[\mathbf{e}_{n}^{(M)}\right]^{\mathcal{H}}\right]\right]\left[\left[\boldsymbol{\Omega}^{n-1} \mathbf{U}\right] \otimes\left[\mathbf{e}_{n}^{(M)} \Theta\right]\right] \\
& =\sum_{n=1}^{M}\left\{\left[\left[[\mathbf{U}]^{\mathcal{H}}\left[\boldsymbol{\Omega}^{n-1}\right]^{\mathcal{H}}\right]\left[\boldsymbol{\Omega}^{n-1} \mathbf{U}\right]\right] \otimes\left[\left[[\Theta]^{\mathcal{H}}\left[\mathbf{e}_{n}^{(M)}\right]^{\mathcal{H}}\right] \mathbf{e}_{n}^{(M)} \Theta\right]\right\} \\
& =\sum_{n=1}^{M}\left\{\mathbf{I}_{2} \otimes \Xi_{n}\right\}=\mathbf{I}_{2 M}
\end{aligned}
$$

where $\Xi_{n}$ is an all-zero square matrix of size $M \times M$ except its value is 1 at the $(n, n)^{t h}$ entry.

\section{DIVERSITY ANALYSIS}

We provide a general diversity analysis for joint 3-D CDC and ECC in this section. We remark that the diversity analysis conducted in [?] only considered 3-D CDC. We assume that one ECC stream is encoded across $K$ STFCs and $N_{a}$ STFBs per STFC with indices $i=i_{1}, \ldots, i_{N_{a}}$. Denote the $i$-th STFB within the $k$-th STFC as $\mathbf{C}^{(i, k)}$, which is formed as

$\mathbf{C}^{(i, k)}=\left[\begin{array}{llll}{\left[\mathbf{C}^{(1, i, k)}\right.} & ]^{\mathcal{T}} & {\left[\mathbf{C}^{(2, i, k)}\right.}\end{array}\right]^{\mathcal{T}} \quad \cdots \quad\left[\begin{array}{ll}\mathbf{C}^{(T, i, k)} & ]^{\mathcal{T}}\end{array}\right]^{\mathcal{T}}$, where $\mathbf{C}^{(t, i, k)}$ has entries $\left[\mathbf{C}^{(t, i, k)}\right]_{a, b}=c_{b, p_{a}^{(i)}}^{(t, i, k)}$, and $c_{m, p_{n_{F}}^{(i)}}^{(t, i, k)}$ is the channel symbol of the $k$-th OFDM block, the $t$-th OFDM block, the $p_{a}^{(i)}$-th subcarrier from the $m$-th transmit antenna, and $p_{a}^{(i)}=p_{1}^{(i)}, \ldots, p_{N_{F}}^{(i)}$ is the subcarrier index for the $i$-th STFB. The received signal corresponding to STFB $\mathbf{C}^{(i, k)}$ can be reformed as

$$
\overline{\mathbf{y}}^{(i, k)}=\sqrt{\frac{\rho}{N_{T}}} \mathbf{M}^{(i, k)} \overline{\mathbf{H}}^{(i, k)}+\overline{\mathbf{v}}^{(i, k)},
$$

where $\overline{\mathbf{y}}^{(i, k)}$ and $\overline{\mathbf{v}}^{(i, k)}$ are the receive signal vector and noise vector, respectively. The CDC based channel symbol matrix $\mathbf{M}^{(i, k)}$ is of size $N_{F} N_{r} T \times N_{F} N_{t} N_{r} T$, and is defined as $\mathbf{M}^{(i, k)}=I_{N_{r}} \otimes\left[\mathbf{M}_{1}^{(i . k)}, \ldots, \mathbf{M}_{N_{t}}^{(i, k)}\right]$, where

$\mathbf{M}_{m}^{(i, k)}=\operatorname{diag}\left(c_{m, p_{1}^{(i)}}^{(1, i, k)}, \ldots, c_{m, p_{N_{F}}^{(i)}}^{(1, i, k)}, \ldots c_{m, p_{1}^{(i)}}^{(T, i, k)}, \ldots, c_{m, p_{N_{F}}^{(i)}}^{(T, i, k)}\right)$.

The equivalent frequency domain channel vector of size $N_{F} N_{t} N_{r} T \times 1$ can be expressed as

$\overline{\mathbf{H}}^{(i, k)}=\left[\begin{array}{l}{\left[\overline{\mathbf{H}}_{1,1}^{(i, k)}\right]^{\mathcal{T}}, \ldots,\left[\overline{\mathbf{H}}_{N_{t}, 1}^{(i, k)}\right]^{\mathcal{T}}, \ldots,\left[\overline{\mathbf{H}}_{1,2}^{(i, k)}\right]^{\mathcal{T}}, \ldots,\left[\overline{\mathbf{H}}_{N_{t}, 2}^{(i, k)}\right]^{\mathcal{T}},} \\ \ldots,\left[\overline{\mathbf{H}}_{1, N_{r}}^{(i, k)}\right]^{\mathcal{T}}, \ldots,\left[\overline{\mathbf{H}}_{N_{t}, N_{r}}^{(i, k)}\right]^{\mathcal{T}}\end{array}\right]^{\mathcal{T}}$

where

$$
\overline{\mathbf{H}}_{m, n}^{(i, k)}=\left[\begin{array}{c}
H_{m, n, p_{1}^{(i)}}^{(1, k)}, H_{m, n, p_{2}^{(i)}}^{(1, k)}, \ldots, H_{m, n, p_{N_{F}}^{(i)}}^{(1, k)}, \ldots, \\
H_{m, n, p_{1}^{(i)}}^{(T, k)}, H_{m, n, p_{2}^{(i)}}^{(T, k)}, \ldots, H_{m, n, p_{N_{F}}^{(i)}}^{(T, k)}
\end{array}\right]^{\mathcal{T}},
$$


and $H_{m, n, p_{n_{F}}^{(i)}}^{(k)}$ is the frequency domain channel gain of the $k$-th OFDM block, the $p_{n_{F}}^{(i)}$-th subcarrier for block between the $m$ th transmit antenna and the $n$-th receive antenna, where $m=$ $1, \ldots, N_{t}$ and $n=1, \ldots, N_{r}$.

Considering a pair of matrices $\mathbf{M}_{(a)}^{(i, k)}$ and $\mathbf{M}_{(b)}^{(i, k)}$ which correspond to two different blocks $\mathbf{C}_{a}^{(i, k)}$ and $\mathbf{C}_{b}^{(i, k)}$, the upper bound for the pairwise error probability between $\mathbf{M}_{(a)}^{(i, k)}$ and $\mathbf{M}_{(b)}^{(i, k)}$ is [?]

$$
\begin{gathered}
\mathbf{P}_{r}\left\{\mathbf{M}_{a}^{(i, k)}-\mathbf{M}_{b}^{(i, k)}\right\} \leqslant\left(\begin{array}{c}
2 r_{(i, k)}-1 \\
r_{(i, k)}
\end{array}\right)\left(\prod_{c=1}^{r_{(i, k)}} \gamma_{c}^{(i, k)}\right)^{-1} \\
\cdot\left(\frac{\rho}{N_{t}}\right)^{-r_{(i, k)}}
\end{gathered}
$$

where $r_{(i, k)}$ has the rank of

$$
\Lambda_{(a, b)}^{(i, k)}=\left(\mathbf{M}_{(a)}^{(i . k)}-\mathbf{M}_{(b)}^{(i . k)}\right) \mathbf{R}_{\overline{\mathbf{H}}^{(i, k)}}\left(\mathbf{M}_{(a)}^{(i . k)}-\mathbf{M}_{(b)}^{(i . k)}\right)^{\mathcal{H}}
$$

and $\mathbf{R}_{\mathbf{H}^{(i, k)}}=\mathbb{E}\left\{\mathbf{H}^{(i, k)}\left[\mathbf{H}^{(i, k)}\right]^{\mathcal{H}}\right\}$ is correlation matrix of $\mathbf{H}^{(i, k)}$, and $\gamma_{c}^{(i, k)}, c=1, \ldots, r_{(i, k)}$ are the non-zero eigenvalues of $\Lambda_{(a, b)}^{(i, k)}$. Denote $\psi_{(b)}^{(i . k)}=\mathbf{M}_{(b)}^{(i, k)}-\mathbf{M}_{(b)}^{(i, k)}$, then $\boldsymbol{\Lambda}^{(i, k)}=\psi_{(a, b)}^{(i . k)} \mathbf{R}_{\overline{\mathbf{H}}^{(i, k)}}\left[\psi_{(a, b)}^{(i . k)}\right]^{\mathcal{H}}$. Also denote

$$
\begin{aligned}
\psi_{(a, b)} & =\operatorname{diag}\left(\psi_{(a, b)}^{(1)}, \ldots, \psi_{(a, b)}^{(K)}\right), \\
\psi_{(a, b)}^{(k)} & =\operatorname{diag}\left(\psi_{(a, b)}^{\left(i_{1}, k\right)}, \ldots, \psi_{(a, b)}^{\left(i_{N a} . k\right)}\right) ; \\
\overline{\mathbf{H}} & =\left[\left[\overline{\mathbf{H}}^{(1)}\right]^{\mathcal{T}}, \ldots,\left[\overline{\mathbf{H}}^{(K)}\right]^{\mathcal{T}}\right]^{\mathcal{T}}, \\
\overline{\mathbf{H}}^{(k)} & =\left[\left[\overline{\mathbf{H}}^{\left(i_{1}, k\right)}\right]^{\mathcal{T}}, \ldots,\left[\overline{\mathbf{H}}^{\left(i_{N_{a}}, k\right)}\right]^{\mathcal{T}}\right]^{\mathcal{T}} ; \\
\mathbf{R}_{\overline{\mathbf{H}}} & =\mathbb{E}\left\{\overline{\mathbf{H}}[\overline{\mathbf{H}}]^{\mathcal{H}}\right\}, \quad \mathbf{M}_{(a)}=\operatorname{diag}\left(\mathbf{M}_{(a)}^{(k)}, \ldots, \mathbf{M}_{(a)}^{(k)}\right),
\end{aligned}
$$

and

$$
\mathbf{M}_{(a)}^{(k)}=\operatorname{diag}\left(\mathbf{M}_{(a)}^{\left(i_{1}, k\right)}, \ldots, \mathbf{M}_{(a)}^{\left(i_{N_{a}}, k\right)}\right) .
$$

The upper bound of the pairwise error probability between $\mathbf{M}_{(a)}$ and $\mathbf{M}_{(b)}$ can now be expressed as

$$
\mathbf{P}_{r}\left\{\mathbf{M}_{(a)}-\mathbf{M}_{(b)}\right\} \leqslant\left(\begin{array}{c}
2 r-1 \\
r
\end{array}\right)\left(\prod_{c=1}^{r} \gamma_{c}\right)^{-1}\left(\frac{\rho}{N_{t}}\right)^{-r}
$$

where $r$ is the rank of $\boldsymbol{\Lambda}_{(a, b)}$, and $\gamma_{c}, c=1, \ldots, r$ are the nonzero eigenvalues of $\boldsymbol{\Lambda}_{(a, b)}$. Note that the upper limit diversity order of this system is

$$
\begin{aligned}
\min \left\{\operatorname{rank}\left(\boldsymbol{\Lambda}_{(a, b)}\right)\right\} & \leqslant K \min \left\{N_{t} N_{r} T(L+1), N_{r} T N_{C}\right\} \\
& \leqslant \operatorname{rank}\left(\mathbf{R}_{\overline{\mathbf{H}}}\right) .
\end{aligned}
$$

For the system under investigation, ECC is applied across the whole data block, and one of the important roles of ECC is to increase distance metrics among different information bit streams: 1) Hamming distance in block ECC; 2) free distance in convolutional or trellis ECC; 3) Euclidean and free distances in trellis coded modulations. The rank $r$ is actually a function of the Hamming or free distance $d$ of ECC, the mapping $\tau$ of the ECC coded bit stream into different STFBs across the whole block, and the mapping $\sigma$ of the ECC coded bit stream into constellation symbols. The system diversity order is thus further bounded by

$$
\begin{aligned}
\min \left\{\operatorname{rank}\left(\boldsymbol{\Lambda}_{(a, b)}\right)\right\} & \leqslant \min \left\{K, d_{\min }\right\} \\
& \cdot \min \left\{N_{t} N_{r} T(L+1), N_{r} T N_{C}\right\} \\
& \leqslant \operatorname{rank}\left(\mathbf{R}_{\overline{\mathbf{H}}}\right)
\end{aligned}
$$

where $d_{\min }$ is the minimum distance of the employed ECC. For block ECC, it refers to Hamming distance; for convolutional codes, it refers to free distance. Let us denote $r=f_{(d, \tau, \sigma)}$. If $r$ and $\prod_{c=1}^{r} \gamma_{c}$ are approximately the same for the same $(d, \tau, \sigma)$, the union bound for the average symbol error rate can be simplified as

$$
\begin{aligned}
\mathbf{P}_{e} \leqslant & \sum_{a} \mathbf{P}_{r}(a) \sum_{b \neq a} \mathbf{P}_{r}\left\{\mathbf{M}_{(a)}-\mathbf{M}_{(b)}\right\} \\
\approx & \sum_{(\tau, d, \sigma)} \frac{W_{(d, \tau, \sigma)}}{N_{B}}\left(\begin{array}{c}
2 f_{(d, \tau, \sigma)}-1 \\
f_{(d, \tau, \sigma)}
\end{array}\right)\left(\prod_{a=1}^{f_{(d, \tau, \sigma)}} \gamma_{c}^{(d, \tau, \sigma)}\right)^{-1} \\
& \cdot\left(\frac{\rho}{N_{t}}\right)^{-f_{(d, \tau, \sigma)}}
\end{aligned}
$$

where $W_{(d, \tau, \sigma)}$ is the number of pairs of $\mathbf{M}_{(a)}$ and $\mathbf{M}_{(b)}$ with the same $(d, \tau, \sigma)$.

In order to demonstrate the relation between the diversity performance and the mapping $\tau$ more precisely, let us assume that the channels are independent over different CDC based STFCs, then

$$
\begin{aligned}
\boldsymbol{\Lambda}_{(a, b)} & =\psi_{(a, b)} \operatorname{diag}\left(\mathbf{R}_{\overline{\mathbf{H}}^{(1)}}, \ldots, \mathbf{R}_{\overline{\mathbf{H}}^{(K)}}\right)\left[\psi_{(a, b)}\right]^{\mathcal{H}} \\
& =\operatorname{diag}\left(\boldsymbol{\Lambda}_{(a, b)}^{(1)}, \ldots, \boldsymbol{\Lambda}_{(a, b)}^{(K)}\right)
\end{aligned}
$$

where $\boldsymbol{\Lambda}_{(a, b)}^{(k)}=\psi_{(a, b)}^{(k)} \mathbf{R}_{\overline{\mathbf{H}}^{(k)}}\left[\psi_{(a, b)}^{(k)}\right]^{\mathcal{H}}$. In what follows, we discuss the mapping $\tau$ of the ECC coded bit stream into different STFBs across the whole block.

1) Case 1: Assume $N_{F} \geqslant N_{T}(L+1)$, and full diversity space-time-frequency CDC, which achieves the upper bound of $\operatorname{rank}\left(\Lambda_{(a, b)}^{(i, k)}\right)$ for any pairs of channel coded streams, is chosen, for each STFB.

Note that

$$
\begin{aligned}
\operatorname{rank}\left(\boldsymbol{\Lambda}_{(a, b)}^{(i, k)}\right) & \leqslant \min \left\{N_{t} N_{r} T(L+1), N_{r} T N_{C}\right\} \\
& \leqslant \operatorname{rank}\left(\mathbf{R}_{\overline{\mathbf{H}}^{(i, k)}}\right) .
\end{aligned}
$$

In this case,

$$
\min \left\{\operatorname{rank}\left(\boldsymbol{\Lambda}_{(a, b)}^{(k)}\right)\right\}=\min \left\{\operatorname{rank}\left(\boldsymbol{\Lambda}_{(a, b)}^{(i, k)}\right)\right\} .
$$

Apparently, increasing the number of STFBs per CDC based STFC to $N_{a}>1$ will not increase the diversity order, which is $\min _{(a, b)}\left\{\operatorname{rank}\left(\boldsymbol{\Lambda}_{(a, b)}^{(k)}\right)\right\}$ for the $k$-th CDC based STFC. However, there might be some coding gain through ECC. In this case, $N_{a}=1$ is the best choice 
for exploiting diversity, i.e., one channel code stream is across multiple STFCs, and the part of the stream with one CDC based STFC is only encoded in one STFB. However, since this may introduce long delay for long channel codes, $N_{a}>1$ may still be a practical choice.

2) Case 2: Assume $N_{F}<N_{T}(L+1)$, and a non-fulldiversity space-time-frequency CDC is chosen for each STFB. In this case, $N_{a}>1$ will increase the diversity order of the $k$-th CDC based STFC.

In the above, we have discussed ECC bit streams across different STFBs. We know that channel codes can be classified as bit-based (e.g., in convolutional codes, BCH codes) or symbolbased (e.g., in Reed Solomon codes). One further issue related to diversity performance for a given channel coded stream concerns the number of units of one channel code stream to be allocated to each STFB. In the case where channels are independent over different CDC based STFCs, and full diversity codes are chosen, the problem is to find the relation between the number of CDC-based STFCs employed and the distances between pairs of channel codes. Ideally, the ECCs operate in channels which are independent for different units. Note that the frequency domain channel gains within one CDC based STFC are correlated as quantified in $\mathbf{R}_{\overline{\mathbf{H}}^{(k)}}$. Thus it is preferable to encode only one unit within one CDC based STFC for a given EC coded stream. Now we have construction the following proposition.

Proposition 1: One STF communications channel is of full rank over space, time, and frequency, and is independent over different STFBs in time. Consider a joint 3-D CDC and channel coding system. The physical dimensions of 3-D CDC STFBs are sufficient to achieve full diversity over space, time, and frequency. The channel coding sequences operate in units (either bits or symbols). There are $N_{u}$ channel coding sequences, and each of them is of length $K$ units and with minimum pairwise distance $d_{\min } \leq K$ units to be encoded into $K$ STFBs. If one STFB only encodes a single unit of each channel coding sequence, the system achieves the diversity order upper bound

$\min \left\{\operatorname{rank}\left(\boldsymbol{\Lambda}_{(a, b)}\right)\right\}=d_{\min } \min \left\{N_{t} N_{r} T(L+1), N_{r} T N_{C}\right\}$. Proof: Note that

$$
\begin{aligned}
& \boldsymbol{\Lambda}_{(a, b)}=\phi_{(a, b)} \operatorname{diag}\left(\mathbf{R}_{\overline{\mathbf{H}}^{(1)}}, \ldots, \mathbf{R}_{\left.\overline{\mathbf{H}}^{(K)}\right)}\left[\phi_{(a, b)}\right]^{\mathcal{H}}\right. \\
& =\operatorname{diag}\left(\boldsymbol{\Lambda}_{(a, b)}^{(1)}, \ldots, \boldsymbol{\Lambda}_{(a, b)}^{(K)}\right), \\
& =\operatorname{diag}\left(\psi_{(a, b)}^{(1)} \mathbf{R}_{\overline{\mathbf{H}}^{(1)}}\left[\psi_{(a, b)}^{(1)}\right]^{\mathcal{H}}, \ldots, \psi_{(a, b)}^{(K)} \mathbf{R}_{\overline{\mathbf{H}}^{(K)}}\left[\psi_{(a, b)}^{(K)}\right]^{\mathcal{H}}\right) .
\end{aligned}
$$

Thus

$$
\operatorname{rank}\left(\boldsymbol{\Lambda}_{(a, b)}\right)=\sum_{k=1}^{K} \psi_{(a, b)}^{(k)} \mathbf{R}_{\overline{\mathbf{H}}^{(k)}}\left[\psi_{(a, b)}^{(k)}\right]^{\mathcal{H}} .
$$

Because each channel coding sequence has minimum pairwise distance $d_{\min } \leq K$ units, there are differences of $d_{\min }$ units for any two different information sequences. Note that one STFB only encodes a single unit of each channel coding sequence, so that there are at least $d_{\min }$ STFBs with different channel coded input for any two different information sequences. Hence,

$$
\begin{aligned}
\min \left\{\operatorname{rank}\left(\boldsymbol{\Lambda}_{(a, b)}\right)\right\} & =d_{\min } \operatorname{rank}\left\{\psi_{(a, b)}^{(1)} \mathbf{R}_{\overline{\mathbf{H}}^{(1)}}\left[\psi_{(a, b)}^{(1)}\right]^{\mathcal{H}}\right\} \\
& =d_{\min } \min \left\{N_{t} N_{r} T(L+1), N_{r} T N_{C}\right\}
\end{aligned}
$$

The coded diversity system described in Proposition 1 actually encodes $N_{u}$ channel coded streams of $K$ units in parallel. If the unit is one bit, we call it a bit-interleaved coded complex diversity coding (BICCDC) based approach. However, in this case, bits are interleaved not simply across different constellation symbols but across different CDC based STFCs. Bitinterleaved coded modulation (BICM) is therefore not appropriate terminology for the proposed system. If the unit is one symbol, we call the corresponding approach symbol-interleaved coded complex diversity coding (SICCDC).

It should be noted that for codes with long block length, both BICCDC and SICCDC may introduce long decoding delays. Therefore, for delay constrained applications, both BICCDC and SICCDC are only suitable for short channel codes. The performance of full diversity SICCDC based STFC will be examined using simulations in Section IV.

\section{NumericAl RESUlts}

Simulation settings are summarized as follows:

1) A convolutional code (with block size of 512 coded bits, code rate $R_{c}=1 / 2$, constraint length 3 , and generator polynomials $(5,7)$ in octal form) is used in Fig. 3; a Reed Solomon code (each RS codeword includes 6 RS information symbols and 2 redundancy symbols, and each RS symbol corresponds to 4 bits) is used in Fig. 4.

2) MIMO frequency selective channel is of order $L=1$ (2 paths), and each path experiences independent Rayleigh fading. Channel power delay profile is assumed to be uniform.

3) $N_{t}=N_{r}=2, N_{F}=4, T=2$, and $N_{C}=32$.

4) the number of STFCs for joint CDC and ECC is $K$, and the number of STFBs within one STFC for one block of joint 3-D CDC and ECC is $N_{a}$.

Fig. 3 shows the performance comparison between ECConly STFCs and joint CDC-ECC STFCs as well as the impact of the parameter $N_{a}$ on the STFC system performance. We apply an iterative MMSE based soft decoding scheme. To maintain the same data rates among ECC-only STFCs and joint CDCECC STFCs, we construct ECC-only STFCs by using identity matrices for $\mathbf{G}_{S T F B}$ in CDC based STFCs. Clearly, joint CDC-ECC STFCs outperform ECC-only STFCs, especially at higher SNRs and when the iterative scheme converges. Consistent with the analysis in Section III, in Fig. 3, the system using full diversity 3-D CDC based STFC with $N_{a}=1$ outperforms that with $N_{a}=4$.

Fig. 4 shows the results of joint full diversity 3-D CDC and ECC with Reed-Solomon (RS) codes. Each RS coded stream is across $N_{a}$ 3-D CDC STFCs, and one RS codeword is only across one STFB within each STFC. The number of RS symbols of one RS codeword within one STFB is $N_{g}$. In the simulations, hard sphere decoding for 3-D CDC STFCs and hard decisions for RS codes are chosen. From Fig. 4, one can see that with the same configurations of RS codewords, STFC using symbol-interleaved coded complex diversity coding (SICCDC), i.e., $N_{g}=1$ significantly outperforms STFC without SICCDC, i.e., $N_{g}=4$. Considering the case when a pair of RS codewords have the minimum distance, i.e., 2 RS symbols, the probability of two different RS symbols being encoded into two different STFBs over 


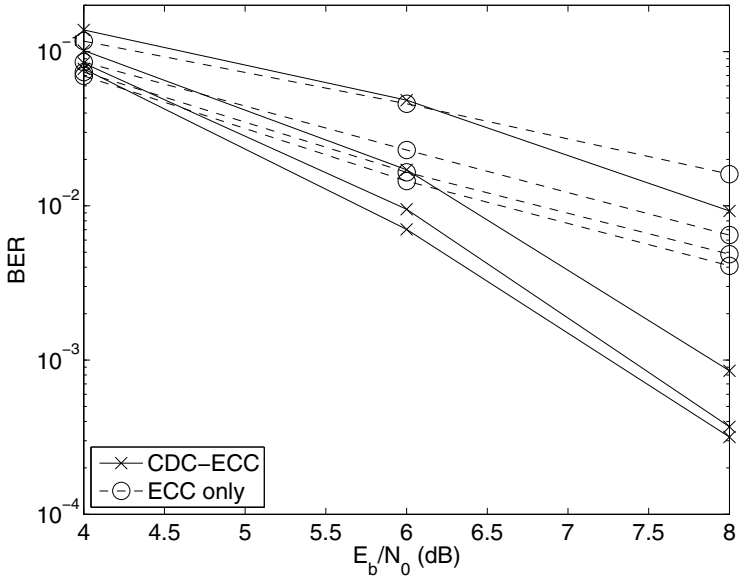

(a) $N_{a}=1$

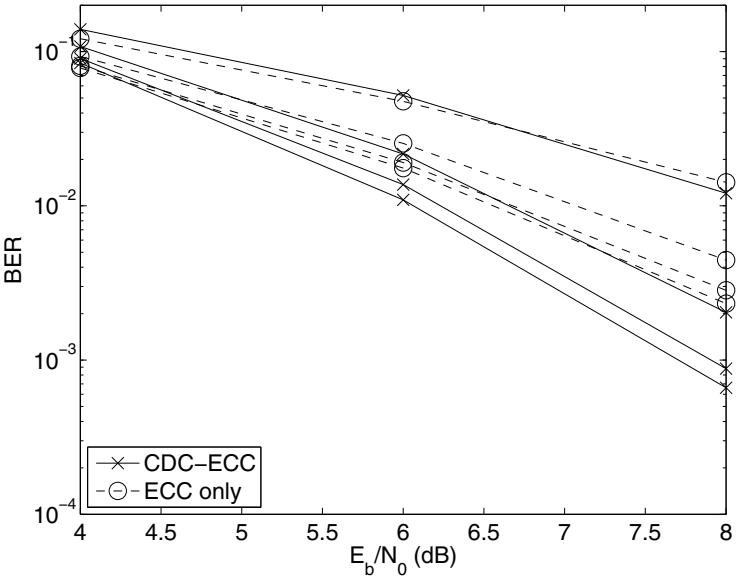

(b) $N_{a}=4$

Fig. 3. STFC performance comparisons between joint CDC-ECC and ECC-only. The top curve represents the first iteration, the bottom curve represents the 4th iteration.

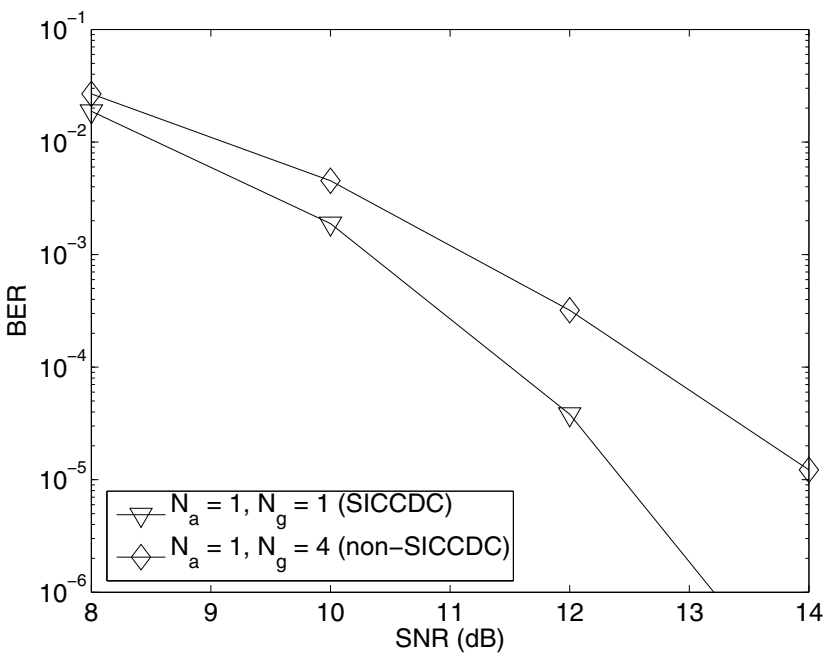

Fig. 4. The effect of using SICCDC on the performance of joint 3-D CDC and RS codes

time is i) 1 in the SICCDC case; ii) $4 / 7$ in the case without using SICCDC. As shown by (15), for the SICCDC case, $\min \left\{\operatorname{rank}\left(\Lambda_{(a, b)}\right)\right\}=2 \min \left\{N_{t} N_{r} T(L+1), N_{r} T N_{C}\right\}$; whereas for the case without SICCDC, $\min \left\{\operatorname{rank}\left(\Lambda_{(a, b)}\right)\right\}=$ $\min \left\{N_{t} N_{r} T(L+1), N_{r} T N_{C}\right\}$. Note that they are the lower bounds, and when a pair of RS codewords differ in more than 2 RS symbols, $\operatorname{rank}\left(\Lambda_{(a, b)}\right)$ may be much higher than $\min \left\{\operatorname{rank}\left(\Lambda_{(a, b)}\right)\right\}$ in the SICCDC case. It is observed from Fig. 4 that the SICCDC with full diversity, proved in Section III, yields superior performance due to its better diversity properties.

\section{Conclusions}

Joint 3-D space-time-frequency CDC and ECC has been investigated in this paper. Our theoretical analysis reveals that by exploiting diversities over all three physical dimensions (spatial, time, and frequency), the joint code design has the potential to achieve a diversity order of $\min \left\{K, d_{\min }\right\} \min \left\{N_{t} N_{r} T(L+1), N_{r} T N_{C}\right\}$, where $N_{t}$ is the number of transmit antennas, $N_{r}$ is the number of receive antennas, $N_{c}$ is the number of subcarriers per antenna, $L$ is the frequency selective channel order between any pair of transmit and receive antennas, $d_{\min }$ is the minimum distance of the employed ECC, and $K$ is the number of 3-D CDC over time. This paper proposes and proves a full diversity construction with joint 3-D CDC and ECC, bit-interleaved coded complex diversity coding and symbol-interleaved coded complex diversity coding. A multi-stream CDC-ECC architecture is also introduced and is shown to have comparable performance to a single-stream system with reduced complexity and decoding latency due to its parallel structure.

\section{REFERENCES}

[1] V. Tarokh, N. Seshadri, A. Calderbank. "Space-time codes for high data rates wireless communications: performance criterion and code construction”. IEEE Trans. on Inform. Theory, vol. 44, pp. 744-765, March 1998.

[2] A. Alamouti. "A simple transmit diversity technqiue for wireless communications". IEEE J. Sel. Areas Commun., vol. 16, no. 8, pp. 1451-1458, Oct. 1998.

[3] V. Tarokh, N. Seshadri, A. Calderbank. "Space-time block coding from orthogonal designs". IEEE Trans. on Inform. Theory, vol. 45, pp. 14561467, July 1999.

[4] Z. Liu, G. Giannakis. "Space-time-frequency coded OFDM over frequency-selective fading channels". IEEE Trans.on Sig. Proc., vol. 50, no. 10, pp. 2465-2476, Oct. 2002.

[5] W. Zhang, X. Xia, P. Ching. "High-rate full-diversity space-timefrequency codes for broadband MIMO block-fading channels". IEEE Trans. on Commun., vol. 55, no. 1, pp. 25-34, Jan. 2007.

[6] J. Wu and S. D. Blostein "High-rate codes over space, time, and frequency ". Proc. IEEE Globecom, pp. 1-6, Nov. 2005.

[7] J. Boutros and E. Viterbo. "Signal space diversity: a power- and bandwidth-efficient diversity technique for the Rayleigh fading channel". IEEE Trans. Inform. Theory, vol. 44, no. 4, pp. 1453-1467, July 1998.

[8] B. Hassibi, B. Hochwald. "High-rate codes that are linear in space and time", IEEE Trans. Inform. Theory, vol. 48, no. 7, pp. 1804-1824, July 2002.

[9] W. Su, Z. Safar, K. Liu. "Towards maximum achievable diversity in space, time, and frequency: performance analysis and code design". IEEE Trans.on Wireless Commun., vol. 4, no. 4, pp. 1847-1857, July 2005.

[10] H. Gamal, A. Hammons. "A new approach to layered space-time coding and signal processing". IEEE Trans. on Information Theory, vol. 47, no. 6, pp. 2321-2334, Sept. 2001

[11] R. Wang, X. Ma, G. Giannakis. "Improving the performance of coded FDFR multi-antenna systems with turbo-decoding". Wireless Communications and Mobile Computing, vol. 4, pp. 711-725, 2004.

[12] S. Siwamogsatham, M. Fitz, J. Grimm. "New view of performance analysis of transmit diversity schemes in correlated Rayleigh fading". IEEE Trans. Inform.Theory, vol. 48, no. 4, pp. 950-956, Apr. 2002. 